\title{
Credits for the illustrations
}

Fig. 1.1 (c) and courtesy of the Department of Physics, Cavendish Laboratory, Cambridge. Photo P24

Fig. 1.2 (c) and courtesy of the Department of Physics, Cavendish Laboratory, Cambridge. Photo P442

Fig. 1.3 C and courtesy of the Oak Ridge National Laboratory, USA.

Fig. 1.4 (C) and courtesy of the CERN, <cdsweb.cern.ch> a crop from photo 6410039

Fig. 1.5 (c) hewat.net/science

Fig. 1.6 (C) 1973 German TV ZDF, "Die stiellen Stars"

Fig. 1.7 (C) "New trends in atomic research and their international significance", Kowarski, L., 1961, Organisation for European Economic Co-operation. European Nuclear Energy Agency, Ed. OEEC-ENEA Paris

Fig. 1.8 (C) 1994 ILL and Jean-Luc Baudet, Grenoble

Fig. 1.9 (C) 2000 ILL and Serge Claisse, Grenoble

Fig. 1.10 (C) and courtesy of the Museum of History of Science and Technology of JINR, $<$ museum.jinr.ru>

Fig. 2.1 (C) 1995, ILL and Serge Claisse, Grenoble.

Fig. 2.2 C ILL, Grenoble

Fig. 2.3 (C) 1980 Photo library < alamy.com>, Photo CR7CWP

Fig. 2.4 (C) and courtesy of the CNRS Grenoble.

Fig. 3.1 C ILL, Layout of spectrometer IN20 from https://www.ill.eu/in20

Fig. 3.2 C ILL, Grenoble

Fig. 3.3 C ILL, Layout of spectrometer IN5 from https://www.ill.eu/in5

Fig. 3.4 (C) ILL, Layout of spectrometer IN15 from https://www.ill.eu/in15

Fig. 4.1 (C) Siemens AG, Munich/Berlin - Siemens Historical Institute beckurts-stiftung.de

Fig. 4.2 Unknown original document.

Fig. 4.3 Left: @ Henri Manuel. Wellcome Collection $<$ wellcomecollection.org $>$. Attribution 4.0 International (CC BY 4.0); right: (C) The Nobel Foundation, unknown photographer. From <en.wikipedia.org>

Fig. 4.4 Unknown original document

Fig. 4.5 Unknown original document

Fig. $5.1 \quad$ (C) 1971 ILL, Grenoble

Fig. $5.2 \quad$ (C) 1971 ILL, Grenoble

Fig. 5.3 (C) 1971 ILL, Grenoble

Fig. 5.4 (C) 1970 ILL, Grenoble

Fig. 5.5 (C) 1998 ILL, Grenoble and C. Designolles

Fig. 5.6 (C) 1970 Le Dauphiné Libéré, Grenoble, 14 Feb. 1970

Fig. 5.7 (C) 2004 ILL, Jean-Luc Baudet

Fig. 5.8 (C) 1979 ILL, Grenoble

Fig. 5.9 "La source de neutrons froids pour le réacteur à haut flux franco-allemand de Grenoble », Ageron P., Ewald R., Harig H.-D. and Verdier J, (1971) Energie Nucléaire, 13, 1-7 
Fig. 5.10 Adapted from the booklet "L'Institut Max von Laue - Paul Langevin", undated but presumably 1971, Presses de Lescuyer at Lyon, France, page 27. See also BIST (Bulletin d'Information Scientifique et Technique du CEA), P. Ageron, 166, Jan. 1972), 17-22

Fig. 5.11 Adapted from the booklet "L'Institut Max von Laue - Paul Langevin", undated but presumably 1971, Presses de Lescuyer at Lyon, France, page 26. See also BIST (Bulletin d'Information Scientifique et Technique du CEA), P. Ageron, 166, Jan. 1972, 17-22

Fig. 5.12 Adapted from the booklet "L'Institut Max von Laue - Paul Langevin", undated but presumably 1971, Presses de Lescuyer at Lyon, France, page 9. See also BIST (Bulletin d'Information Scientifique et Technique du CEA), P. Ageron, 166, Jan. 1972, 17-22

Fig. 5.13 (C) 1984 ILL, Grenoble

Fig. 5.14 Photo from Bernard Jacrot's archives.

Fig. 5.15 From Ipoustéguy

Fig. 5.16 (C) 1989 "Ipoustéguy", Pierre Gaudibert, Ed. Cercle d'Art, Paris (1989) p 139

page 97 Alan Daramsy and Thomas Hansen, (C2014 ILL and Max Alexander

page 97 Oscar Ramon Fabelo Rosa, (C2016 ILL and Ecliptique <ecliptique.com>, Laurent Thion

page 97 Matthew Blakeley, (2015 ILL and Robert Cubitt

page 97 Thilo Pirling and Sofiane Terzi, (02016 ILL and Ecliptique

page 99 C2009 ILL and Artechnique

page 99 Daniela Russo, (C2012 ILL and Ecliptique

page 99 Sacha Ivanov and Eric Bourgeat Lami, (C2016 ILL and Ecliptique

page 99 Peter Falus and Ingo Hoffmann, (C2017 ILL and Ecliptique

page 101 Richard Campbell, C2016 ILL and Ecliptique

page 101 Valery Nesvizhevsky, @2009 ILL and A. Chézière

page 101 Tobias Jenke and Erwin Jericha (02016 ILL and Ecliptique

page 101 Thomas Materna, C2015 ILL and Ulli Köster

Fig. 6.1 C ILL, Grenoble

Fig. 6.2 (C) ILL, Grenoble

Fig. 7.1 1968 ILL and Studio Givet, Grenoble

Fig. 7.2 (C) 2013 ESRF and Jean Moulin

Fig. 7.3 (C) 2001 ILL, Millennium Symposium 2001

Fig. 7.4 C 2003 ILL, Rapport de sureté, Dec 2003, Fig. 23.24.1

Fig. 8.1 (C) 1994 ILL and Jean-Louis Baudet

Fig. 9.1 (C) ILL, Grenoble

Fig. 11.1 (C) 2006 ILL and P.-Y. Meynent

Fig. 11.2 (C) 2018 ILL and A. Filhol

Fig. 11.3 (C) 2008 ILL and Serge Claisse

Fig. 11.4 (C) 2018 ILL, Grenoble

Fig. 11.5 (C) 2019 ILL and Ecliptique

Fig. 11.6 (C) 2003 ILL, Grenoble

Fig. 11.7 (C) 2011 ILL, Grenoble

Fig. 11.8 (C) 2016 ILL and Ecliptique

Fig. 11.9 (C) 2018 ILL and renishaw.com

Fig. 11.10 (C) 2014 Serebrov A., Petersburg Nuclear Physics Institute

Fig. 11.11 @ 2018 ILL and G. Cicognani 
